\title{
Hawking Temperature of the Reissner-Nordstrom-Vaidya Black Hole
}

\author{
Triyanta $^{1,2}$ \& Anike N. Bowaire ${ }^{1}$ \\ ${ }^{1}$ Division of Theoretical High Energy Physics and Instrumentation, \\ Faculty of Mathematics and Natural Sciences, Institut Teknologi Bandung \\ Jalan Ganesha No. 10 Bandung 40132 Indonesia \\ ${ }^{2}$ Indonesian Center for Theoretical and Mathematical Physics, \\ Faculty of Mathematics and Natural Sciences, Institut Teknologi Bandung \\ Jalan Ganesha No. 10 Bandung 40132 Indonesia \\ Email: triyanta@fi.itb.ac.id
}

\begin{abstract}
We have derived the Hawking temperature of the ReissnerNordstrom-Vaidya black hole by the use of both the radial null geodesic and the complex path methods. We have obtained that the mass dependence of the temperature in the Reissner-Nordstrom-Vaidya black hole is not as simple as that in the Schwarzschild black hole, but still we regain the conclusion, as in the case of the Schwarzschild black hole, that a higher Hawking temperature corresponds to a lower mass of a black hole.
\end{abstract}

Keywords: black hole horizon; charged black hole; complex path; extreme ReissnerNordstrom-Vaidya black hole; Hamilton-Jacobi equation; Hawking temperature; radial null geodesic; Reissner-Nordstrom-Vaidya black hole.

\section{$1 \quad$ Introduction}

A black hole is a very dense object that, classically, particles and light cannot propagate away from it [1-3]. Quantum mechanically, however, there is a possibility that one of a particle production pair in a black hole is able to tunnel the gravitational barrier and escapes the black hole's horizon. Thus, a black hole is not really black; it can radiate or evaporate particles. Such radiation was shown for the first time by Steven Hawking in 1974 [4,5]. As it radiates, a black hole has a non-zero temperature, called the Hawking temperature. In deriving the temperature, Hawking considered the Schwarzschild black hole, a neutral black hole that has no angular momentum. He obtained that the Hawking temperature of the Schwarzschild black hole is $T_{H}=h c / 16 \pi^{2} k M$ where $k$ is the Boltzmann's constant and $M$ is the mass of the black hole [4]. It turns out that the Hawking temperature is inversely proportional to the mass of the black hole. Thus, the possibility for a particle to escape from a heavier black hole is smaller than from a lighter one. Recently, some models of black hole radiation or some ways of deriving the black hole's temperature have been developed, including the radial null geodesic method and the complex path method or the Hamilton-

Received December $17^{\text {th }}, 2012$, Revised April 10 ${ }^{\text {th }}, 2013$, Accepted for publication April 10 ${ }^{\text {th }}, 2013$.

Copyright (c) 2013 Published by ITB Journal Publisher, ISSN: 2337-5760, DOI: 10.5614/j.math.fund.sci.2013.45.2.2 
Jacobi method [2,3,6-8]. Hawking radiation is a hot topic that has been discussed in a number of papers on gravity, including some that were published very recently [9-16]. Previously, we have derived the Hawking temperature of a Vaidya black hole by the use of both methods mentioned before [17]. A Vaidya black hole is a black hole of which the mass depends on radius and time, thus, in the case of the radiation aspect of black holes, it is more realistic as compared to a static one. In this research we considered the Hawking temperature of a Reissner-Nordstrom-Vaidya black hole, i.e. a Vaidya black hole that has a nonzero electric charge. The black hole is specified by the following spacetime interval [18]:

$$
\begin{aligned}
& d s^{2}=-F d t^{2}+F^{-1} d r^{2}+r^{2} d \Omega^{2}, \\
& F=1-\frac{2}{r}(M+f)+\frac{e^{2}}{r^{2}}, d \Omega^{2}=d \theta^{2}+\sin ^{2} \theta d \varphi^{2} .
\end{aligned}
$$

$M$ and $e$ correspond to the mass and charge of a spherically symmetric black hole and $f$ is an arbitrary function of mass $M$ and charge $e, f=f(M, e)$. The above metric is the transformed form of the original one, i.e.

$$
d s^{2}=-F d u^{2}+2 d u d r+r^{2} d \Omega^{2} .
$$

Here $u$ represents a time variable. This form of metric is not applicable for the complex path method since it does not give information on the velocity of a particle moving out. Accordingly, we will not consider this metric for our calculations (especially for the complex path method).

Note that the metric (1) reduces to the Reissner-Nordstrom metric when $f=0$ and reduces to the Schwarzschild metric when both $f$ and $e$ are set to vanish. The Reissner-Nordstrom-Vaidya metric has two singularity (corresponding to $F=0$ ) coordinates

$$
r_{h}=(M+f) \pm \sqrt{(M+f)^{2}-e^{2}} .
$$

Note that single singularity corresponds to a neutral, $e=0$, black hole. Without changing physical content we can define the transformation for the time coordinate

$$
d t \rightarrow d t-\sqrt{1-F^{-1}} d r
$$

giving spacetime interval

$$
d s^{2}=-F d t^{2}+\left(1-F+F^{-1}\right) d r^{2}+2 F \sqrt{1-F^{-1}} d r d t+r^{2} d \Omega^{2} .
$$


In the following two sections, we will utilize this metric to compute the Hawking temperature with the radial null geodesic method and the complex path method. The final section is devoted to the conclusions.

\section{Radial Null Geodesic Method}

The Hawking temperature describes the appearance of black hole radiation. Quantum mechanically, this shows a tunneling effect surrounding the black hole. Since the tunneling probability of a particle corresponds to its action,

$$
\Gamma \propto \exp \left(-\frac{2}{\hbar} \operatorname{Im} S\right)
$$

and the action relates to energy and thus temperature, the corresponding temperature will then define the Hawking temperature. Accordingly we should consider the action to obtain the Hawking temperature. One way to do this is to consider the radial null geodesic condition $d s^{2}=d \Omega^{2}=0$. This condition is equivalent to considering to a two-dimensional $(t-r)$-case. This can be understood since for the spherical symmetric black hole, as in our case, the propagation of particles crossing the gravitational barrier is mostly along the radial direction. Applying this condition to the above metric, one has

$$
\frac{d r}{d t}=\frac{-F \sqrt{1-F^{-1}} \pm 1}{1-F+F^{-1}}=\frac{F}{F \sqrt{1-F^{-1}} \pm 1} .
$$

Note that the second equality comes from the fact that multiplication between the LHS numerator and RHS denominator is equal to that between the RHS numerator and LHS denominator. Let us look at a region around the black hole's horizon by expanding the function $F$ around the horizon's radius $r_{h}$ :

$$
F(r, t) \approx F^{\prime}\left(r_{h}, t\right)\left(r-r_{h}\right)+0\left(r-r_{h}\right)^{2} .
$$

In the above, we take, for simplicity, only the first term. The prime (dot) sign defines the derivative with respect to radial coordinate $r$ (time $t$ ). The velocity around the horizon is

$$
\dot{r}\left(r_{h}, t\right) \approx \pm F^{\prime}\left(r_{h}, t\right)\left(r-r_{h}\right) .
$$

The plus sign corresponds to the velocity of a particle going out from $r_{\text {in }}$ to $r_{\text {out }}$ crossing the barrier near the horizon, while the minus sign defines the velocity of a particle near the horizon moving into the black hole.

The radial part of the action of a particle is defined as 


$$
S_{r}=\int_{r_{\text {in }}}^{r_{\text {out }}} p_{r} d r=\int_{r_{\text {in }}}^{r_{\text {out }}} \int_{0}^{p_{r}} d p_{r} d r=\int_{r_{\text {in }}}^{r_{\text {out }}} \int_{0}^{H} \frac{d H}{\dot{r}} d r, \quad \dot{r}=\frac{d r}{d t}=\frac{d H}{d p_{r}} .
$$

In the above $p_{r}$ is the radial momentum and $H$ is the Hamiltonian. Recalling (8) for outgoing particles, one has

$$
S_{r} \approx \int_{r_{\text {in }}}^{r_{\text {out }}} \int_{0}^{E} \frac{d H}{F^{\prime}\left(r_{h}, t\right)\left(r-r_{h}\right)} d r .
$$

The integration is easily computed. Note that the $r$ integration can be computed by the use of the residue method. We obtain

$$
\operatorname{Im} S_{r} \approx \frac{\pi E(t)}{F^{\prime}\left(r_{h}, t\right)} \text {. }
$$

Due to transformation (4), the time coordinate becomes imaginary at positions where $F<1$. Accordingly, the temporal part of the action, $-E \Delta t$ also contains imaginary values. For the case of the Schwarzschild background, the imaginary component of the temporal part of the action can be obtained through transformation from Schwarzschild coordinates to Kruskal-Szekeres coordinates [19]. It is shown in [19] that the time shift $\Delta t$ has the form of $-i 2 \pi M$. It turns out that the value $2 M$ corresponds to the inverse of $d F / d r$ at the horizon where $F$ for the Schwarzschild background is equal to $1-2 M / r$. Thus, in our case we expect that $-E \Delta t$ equals $i \pi E /\left.(d F / d r)\right|_{\text {horizon }}$ with $F$ is given by Eq. (1). Inclusion of this contribution to the action results in multiplying the right-hand side of Eq. (12) with a factor of 2 :

$$
\operatorname{Im} S \approx \frac{2 \pi E(t)}{F^{\prime}\left(r_{h}, t\right)} .
$$

Finally, equalizing the tunneling probability (6) with the Boltzmann factor $\exp (-E / k T)$ we obtain the Hawking temperature

$$
T_{H}=\frac{\hbar F^{\prime}\left(r_{h}, t\right)}{4 \pi k} \text {. }
$$

The same result can be obtained when we utilize metric (2). In this case the temporal part of the action is real and thus does not contribute to the Hawking temperature.

In the following we derive the Hawking temperature by the use of the second method. 


\section{Complex Path Method}

In this method we work in scalar field theory in which particles (assumed to be mass-less) that radiate out obey the Klein-Gordon's equation with the above metric as the background. We assume that the black hole absorbs almost all particles that move into it, meaning that the probability of ingoing particles is almost unity. In addition, the probability of particles being created within the black hole and radiating out from the black hole is assumed to be equal to $\exp (-E(t) / k T)$. By deriving the action $S(r, t)$ through the Hamilton-Jacobi equation and writing the wave function $\phi$ as $\exp (-2 \pi i S(r, t) / h)$, the Hawking temperature may be obtained.

Let us start with the mass-less Klein-Gordon's equation:

$$
\partial_{\mu}\left[g^{\mu \nu} \sqrt{-g} \partial_{v} \phi\right]=0 .
$$

As a Reissner-Nordstrom-Vaidya black hole is spherically symmetric, the Hawking temperature may be thought of as corresponding to radial radiation. Accordingly, the above equation reduces to a two-dimensional $(r-t)$ problem. For the Reissner-Nordstrom-Vaidya metric (5), the above equation is equivalent to

$$
\begin{aligned}
0= & \partial_{t}\left[g^{t t} \sqrt{-g} \partial_{t} \phi+g^{t r} \sqrt{-g} \partial_{r} \phi\right]+\partial_{r}\left[g^{r t} \sqrt{-g} \partial_{t} \phi+g^{r r} \sqrt{-g} \partial_{r} \phi\right] \\
= & \dot{F}\left(1+F^{-2}\right) \partial_{t} \phi+\left(-1+F-F^{-1}\right) \partial_{t}^{2} \phi+\dot{F}\left(1-F^{-1}\right)^{1 / 2} \partial_{r} \phi+ \\
& \frac{1}{2}\left(1-F^{-1}\right)^{-1 / 2} F^{-1} \dot{F} \partial_{r} \phi+2 F\left(1-F^{-1}\right)^{1 / 2} \partial_{t} \partial_{r} \phi+\left(1-F^{-1}\right)^{1 / 2} F^{\prime} \partial_{t} \phi+ \\
& \frac{1}{2}\left(1-F^{-1}\right)^{-1 / 2} F^{-1} F^{\prime} \partial_{t} \phi+F^{\prime} \partial_{r} \phi+F \partial_{r}{ }_{r} \phi .
\end{aligned}
$$

In the above, the prime sign defines the derivative with respect to radial coordinate $r$ and the dot sign defines the derivative with respect to time $t$. In terms of the action:

$$
\begin{aligned}
0= & \dot{F}\left(1+F^{-2}\right)(-i \hbar) \partial_{t} S+\left(-1+F-F^{-1}\right)\left(-\left(\partial_{t} S\right)^{2}-i \hbar \partial_{t}{ }^{2} S\right)+ \\
& \dot{F}\left(1-F^{-1}\right)^{1 / 2}(-i \hbar) \partial_{r} S+\frac{1}{2}\left(1-F^{-1}\right)^{-1 / 2} F^{-1} \dot{F}(-i \hbar) \partial_{r} S+ \\
& 2 F\left(1-F^{-1}\right)^{1 / 2}\left(-\left(\partial_{t} S\right)\left(\partial_{r} S\right)-i \hbar \partial_{t} \partial_{r} S\right)+\left(1-F^{-1}\right)^{1 / 2} F^{\prime}(-i \hbar) \partial_{t} S+ \\
& \frac{1}{2}\left(1-F^{-1}\right)^{-1 / 2} F^{-1} F^{\prime}(-i \hbar) \partial_{t} S+F^{\prime}(-i \hbar) \partial_{r} \phi+F\left(-\left(\partial_{r} S\right)^{2}-i \hbar \partial_{r}{ }_{r}^{2} S\right) .
\end{aligned}
$$

As the Planck constant is very small, we may approximate to non Planck constant terms. We have

$$
\partial_{r} S=\frac{-F \sqrt{F^{2}-F} \pm F}{F^{2}} \partial_{t} S .
$$


Now use the above equation to obtain the action function. In the standard Hamilton-Jacobi method, $S(r, t)$ can be written into two parts: the time part which has the form of $E t$ and the radius part $R(r)$. However, allowing the energy to vary with time, the action should have a more general form:

$$
S(r, t)=\int_{0}^{t} E\left(t^{\prime}\right) d t^{\prime}+R(r, t) .
$$

Insertion of Eq. (19) into Eq. (18) gives

$$
\partial_{r} S=\partial_{r} R=\frac{-F \sqrt{F^{2}-F} \pm F}{F^{2}}\left(E(t)+\partial_{t} R\right) .
$$

As a function of $r$ and $t$ and recalling Eqs. (7) and (19), the function $R(r, t)$ fulfills

$$
\begin{aligned}
\frac{d R(r, t)}{d r} & =\frac{\partial R(r, t)}{\partial r}+\frac{d t}{d r} \frac{\partial R(r, t)}{\partial t} \\
& =\frac{-\sqrt{F^{2}-F} \pm 1}{F}\left(E(t)+\partial_{t} R\right)+\frac{F \sqrt{1-F^{-1}} \pm 1}{F} \partial_{t} R \\
& =\frac{-\sqrt{F^{2}-F} \pm 1}{F} E(t)+\frac{ \pm 2}{F} \partial_{t} R
\end{aligned}
$$

Considering a slowly varying $R$, the second term may be neglected. The function $R$ in the near horizon may then be obtained by integrating the above equation with respect to $r$, after recalling (8):

$$
R(r, t)= \pm E(t) \int d r \frac{1}{F(r, t)} \approx \pm E(t) \frac{\pi i}{F^{\prime}(r, t)} .
$$

Accordingly, the action has the form

$$
S\left(r_{h}, t\right) \approx \int_{0}^{t} E\left(t^{\prime}\right) d t^{\prime} \pm \frac{\pi i E(t)}{F^{\prime}\left(r_{h}, t\right)} .
$$

In the tunneling process, the particle's energy is less than the barrier potential. Accordingly, the particle's momentum is imaginary, resulting in the imaginary of the action function. Thus we may write $\int E d t$ in the above as $i \operatorname{Im}\left(\int E d t\right)$. Thus, the probability of particles moving in and moving out, respectively, becomes 


$$
\begin{aligned}
& P_{\text {in }}(r, t)=\exp \left[\frac{2}{\hbar}\left(\operatorname{Im} \int E\left(t^{\prime}\right) d t^{\prime}+E(t) \frac{\pi}{F^{\prime}(r, t)}\right)\right], \\
& P_{\text {out }}(r, t)=\exp \left[\frac{2}{\hbar}\left(\operatorname{Im} \int E\left(t^{\prime}\right) d t^{\prime}-E(t) \frac{\pi}{F^{\prime}(r, t)}\right)\right] .
\end{aligned}
$$

Since we assume that almost all ingoing particles are trapped, we have $P_{\text {in }}$ equals to unity and thus

$$
\operatorname{Im} \int E\left(t^{\prime}\right) d t^{\prime}=-E(t) \frac{\pi}{F^{\prime}(r, t)}
$$

giving

$$
P_{\text {out }}(r, t)=\exp \left[\frac{2}{\hbar}\left(-E(t) \frac{2 \pi}{F^{\prime}(r, t)}\right)\right] .
$$

Equalizing the above with $\exp (-E(t) / k T)$ we get the Hawking temperature

$$
T_{H}=\frac{\hbar F^{\prime}(r, t)}{4 \pi k} .
$$

This result agrees with (14) derived by the first method. Thus we conclude that Eq. (27) is the Hawking temperature for the Reissner-Nordstrom-Vaidya black hole.

Let us now look at the explicit form of $F$ (Eq. (1)). We have the explicit form of the Hawking temperature for the Reissner-Nordstrom-Vaidya black hole:

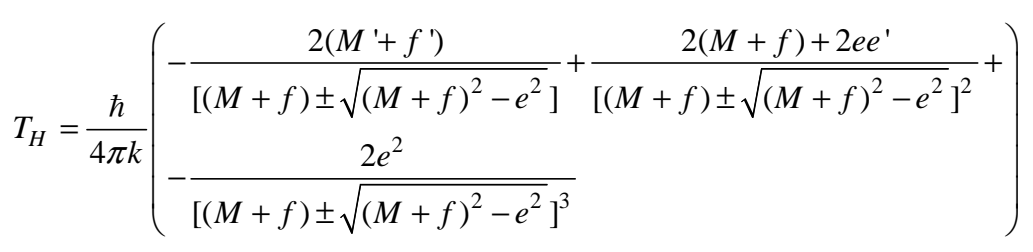

In the \pm sign, plus (minus) corresponds to a larger (smaller) radius of the singularity. Thus we have different Hawking temperatures for different horizons: the larger radius has a lower temperature, and vice versa.

The temperature difference between both horizons is

$$
\Delta T_{H}=\frac{\hbar}{4 \pi k e^{4}}\left(-4\left(M^{\prime}+f^{\prime}\right) e^{2}+8(M+f) e e^{\prime}+4 e^{2}-8(M+f)^{2}\right) \sqrt{(M+f)^{2}-e^{2}}
$$

For the case when $f=e=0$, i.e. for the case of a Schwarzschild black hole, Eq. (28) reads 


$$
T_{H}=\left\{\begin{array}{cc}
\frac{h}{8 \pi^{2} k}\left(-\frac{M^{\prime}}{M}+\frac{1}{2 M}\right) & M=M(r) \\
\frac{h}{16 \pi^{2} k M} & M \neq M(r)
\end{array}\right.
$$

Note that the second equation is in accordance with Hawking's result. This is understood as the case $f=e=0$, and $M$ is $r$-independent corresponds to the Schwarzschild black hole. Note also that this black hole has only one horizon radius, corresponding to the plus sign in Eq. (28), and thus Eq. (29) is not applicable.

Finally, the Hawking temperature for the case of an extreme ReissnerNordstrom-Vaidya black hole where $M=e$, is

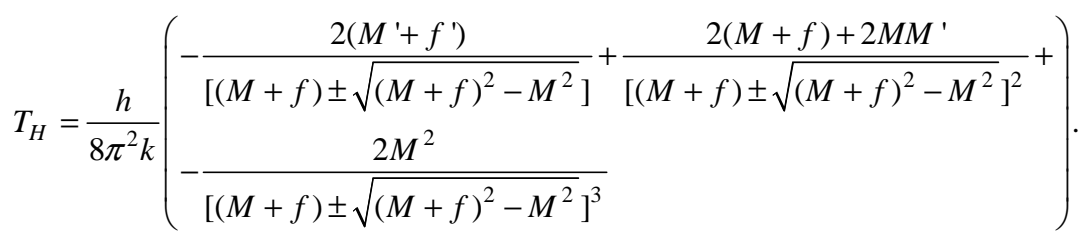

Note that the above temperature vanishes when $f=0$. We see complexities of the mass dependence in the Eqs. (28) and (31). Since the mass in the numerator has lower power compared to that in the denominator, we conclude that a more massive black hole has a lower Hawking temperature compared to a less massive one.

\section{Conclusion}

We have derived the Hawking temperature for a Reissner-Nordstrom-Vaidya black hole with two different methods. The mass dependence of the temperature is not as simple as for the temperature of a Schwarzschild black hole. This is due to the complexity of the Reissner-Nordstrom-Vaidya metric. However, the general conclusion that heavier black holes have a lower Hawking temperature is guaranteed.

\section{Acknowledgments}

This research was supported by the Riset dan Inovasi KK Institut Teknologi Bandung.

\section{References}

[1] Wald, R.M., General Relativity, The Univ. of Chicago Press, 1984. 
[2] Parikh, M.K. \& Wilczek, F., Hawking Radiation as Tunneling, Phys. Rev. Lett., 85, pp. 5042-5045, [arXiv:hep-th/9907001v3], 2000.

[3] Parikh, M.K., Membrane Horizons: The Black Hole's New Clothes, Ph.D. Thesis, Princeton University, Princeton, NJ [arXiv:hep-th/9907002], 1998.

[4] Hawking, S.W., Particle Creation by Black Holes, Comm. Math. Phys., 43, pp. 199-220, 1975.

[5] Wald, R.M., Quantum Field Theory in Curved Spacetime and Black Hole Thermodynamics, The Univ. of Chicago Press, 1994.

[6] Srinivasan, K. \& Padmanabhan, T., Particle Production and Complex Path Analysis, Phys. Rev., D 60, pp. 024007(1-20) [arXiv:hepth/9812028v1], 1999.

[7] Shankaranarayanan, S., Padmanabhan, T. \& Srinivasan, K., Hawking Radiation in Different Coordinate Settings: Complex Path Approach, Class. Quant. Grav., 19, pp. 2671-2688 [arXiv:gr-qc/0010042], 2002

[8] Rahman, M.A. \& Hossain, M.I., Hawking Radiation of Schwarzschild-de Sitter Black Hole by Hamilton-Jacobi Method, Phys. Lett., B 712, pp. 15, 2012.

[9] Modak, S.K. \& Singleton, D., Hawking Radiation as a Mechanism for Inflation, Int. J. Mod. Phys., D21, pp. 1242020, 2012.

[10] Fischetti, S. \& Marolf, D., Flowing Funnels: Heat Sources for Field Theories and the AdS_3 Dual of CFT_2 Hawking Radiation, Class. Quantum Grav., 29, pp. 105004, 2012.

[11] Smolyaninov, I.I., Hwang, E. \& Narimanov, E., Hyperbolic Metamaterial Interfaces: Hawking Radiation from Rindler Horizons and the "End of Time", Phys. Rev., B 85, pp. 235122, 2012.

[12] Jannes, G., Hawking Radiation of $E<M$ Massive Particles in The Tunneling Formalism, JETP Lett., 94, pp. 18-21, 2011.

[13] Barbado, L.C., Barceló, C. \& Garay, L.J., Hawking Radiation as Perceived by Different Observers, Class. Quant. Grav., 28, pp. 125021, 2011.

[14] Kaeonikhom, C., Singleton, D., Sushkov, S.V. \& Yongram, N., Dynamics of Dirac-Born-Infeld Dark Energy Interacting with Dark Matter, Phys. Rev., D86, pp. 124049, 2012.

[15] Modak, S.K. \& Singleton, D., Inflation with A Graceful Exit and Entrance Driven by Hawking Radiation, Phys. Rev., D86, pp. 123515, 2012.

[16] Rad, N. \& Singleton, D., A Test of The Circular Unruh Effect Using Atomic Electrons, Eur. Phys. J., D66, pp. 258, 2012.

[17] Siahaan, H.M. \& Triyanta, Semiclassical Methods for Hawking Radiation from a Vaidya Black Hole, Int. J. of Mod. Phys., A 25(01), pp. 145-153, 2010. 
[18] Ibohal, Ng. \& Kapil, L., Charged Black Holes in Vaidya Backgrounds: Hawking Radiation, Int. J. of Mod. Phys., D 19(04), pp. 437-464, [arXiv:gr-qc/1001.2616], 2010.

[19] Akhmedov, E.T., Pilling, T. \& Singleton, D., Subtleties in the QuasiClassical Calculation of Hawking Radiation, Int. J. of Mod. Phys., D 17 (13 \& 14), pp. 2453-2458, 2008. 